\title{
MODELLING OF CHARGE CARRIER MOBILITY FOR TRANSPORT BETWEEN ELASTIC POLYACETYLENE-LIKE POLYMER NANORODS
}

\author{
"MIROSLAV MENŠÍK, SHIH-JYE SUN*, PETR TOMAN, KAREL KRÁL** \\ Institute of Macromolecular Chemistry, Academy of Sciences of the Czech Republic, \\ Heyrovsky Sq. 2, 16206 Prague 6, Czech Republic \\ *Department of Applied Physics, National University of Kaohsiung, \\ Kaohsiung 811, Taiwan \\ **Institute of Physics, Academy of Sciences of the Czech Republic, \\ Na Slovance 2, 18201 Praha 8, Czech Republic \\ \#E-mail: mensik@imc.cas.cz
}

Submitted September 2, 2016; accepted December 7, 2016

\begin{abstract}
Keywords: Charge carrier mobility, Polymers, Electron-phonon coupling, Polyacetylene
A quantum model solving the charge carrier mobility between polyacetylene-like polymer nanorods is presented. The model assumes: a) Quantum mechanical calculation of hole on-chain delocalization involving electron-phonon coupling leading to the Peierls instability, b) Hybridization coupling between the polymer backbone and side-groups (or environmental states), which act as hole traps, and c) Semi classical description of the inter-chain hole transfer in an applied voltage based on Marcus theory. We have found that mobility resonantly depends on the hybridization coupling between polymer and linked groups. We observed also non-trivial mobility dependences on the difference of energies of the highest occupied molecular orbitals localized on the polymer backbone and side-groups, respectively, and hole concentration. Those findings are important for optimization of hybrid opto-electronic devices.
\end{abstract}

\section{INTRODUCTION}

Organic semiconductors keep going on attracting our attention due to their some very good intrinsic properties allowing device performance tuning as well as fabrication for industry concerns [1]. Meanwhile, some existing limitations in their properties reduce the performances of the device applications, e.g., low carrier mobility and short lifetime [2-3]. In general, in ideal molecular devices people suppose the carriers transport along the backbone of the molecular chains [4]. Actually, the disordered structures of polymers make this ideal limit hard to achieve because the structures are rather twining [5]. For real systems the charge transport in the bulk is rather controlled by the hopping mechanism between polymer chains [6] inside segments of „crystalline-like“ domains.

In this article we present a study of charge carrier mobility in acetylene-like systems [7] within the SuSchrieffer-Heeger (SSH) model [8]. We assume that for each monomer unit (site) there is just one unpaired electron. For neutral polymer chain consisting of $\mathrm{N}$ sites there are $2 \mathrm{~N}$ available states due to the spin degeneracy, but only $\mathrm{N}$ electrons. Therefore, in the ground state the band is half-filled. Moreover, electron-phonon coupling breaks the symmetry of the length of bonds leading to the Peierls instability (bond alternation). Our model additionally assumes that each monomer unit is coupled to environment, (e.g., side groups) which allows a charge transfer, i.e., hybridization of the backbone [9]. Those environmental states can also represent surface states linked to charge transporting channel in optoelectronic devices. The main goal of this article is to study the influence of this hybridization coupling on charge carrier mobility in such polyacetylene-like systems as it provides insight to the basic processes in functionalized optoelectronic devices.

\section{MODEL}

Inter-chain transport of charges

The mobility $\mu$ of the charge carrier in a polymer crystal domain consisting of $\pi$-stacked layers (lamellas) as shown in Figure 1 is dominantly controlled in the bulk by the hopping between chains in the longest direction and therefore it is calculated in the following way. We have taken into account a fixed number (in our case 20) of polymer lamellas in a "box" (corresponding to an elbow of polymer chains in Fig. 1) and for the applied voltage as indicated in Figure 1 we have calculated self-consistently charge occupation probabilities of all eigenstates of all chains as well as charge transition rates between all chains. At the boundaries of the "box" we have assumed periodic boundary conditions. Then, the charge mobility was directly obtained from the formula 


$$
\mu=\frac{d}{E} \Sigma_{\mathrm{A}, \mathrm{B}}\left(T_{\mathrm{AB}}-T_{\mathrm{BA}}\right),
$$

where $d$ and $E$ are the space distance between two nearest neighbor polymer layers and the intensity of the applied electric field, respectively; $T_{A B}$ and $T_{B A}$ are transition rates from layer $\mathrm{A}$ to the nearest neighbor layer $B$ and vice versa. These transition rates form an ensemble of the transition probabilities between occupied and un-occupied states belonging to two nearest neighbor polymer layers, respectively. Thus the transition rate can be written as

$$
T_{\mathrm{AB}}=\Sigma_{\alpha \in \mathrm{A}, \beta \in \mathrm{B}} f\left(E_{\alpha}\right)\left(1-f\left(E_{\beta}\right)\right) v_{\alpha \beta},
$$

where $E_{\alpha}$ (or $f\left(E_{\alpha}\right)$ ) is the electron energy level (or charge occupation probability, respectively) of the $\alpha$-th state of the polymer chain in the layer $\mathrm{A} ; v_{\alpha \beta}$ is the charge exchange rate from the state $\alpha$ of the polymer chain in layer A to the state $\beta$ of the polymer chain in layer B. For the calculation of the charge exchange rate $v_{\alpha \beta}$ we employ the Marcus theory and its formula is written in the form

$$
v_{\alpha \beta}=\frac{J_{\alpha \beta}^{2}}{\hbar} \sqrt{\frac{\pi}{\lambda k_{\mathrm{B}} T}} \exp \left\{-\frac{\left(E_{\alpha}-E_{\beta}-\lambda\right)^{2}}{4 \lambda k_{\mathrm{B}} T}\right\} .
$$

In this formula the charge exchange rate involves some quantum parameters: a) $\lambda$ is the reorganization energy given by the difference of equilibrium position of respective potentials for donor and acceptor states. This includes all vibrational modes not introduced below as

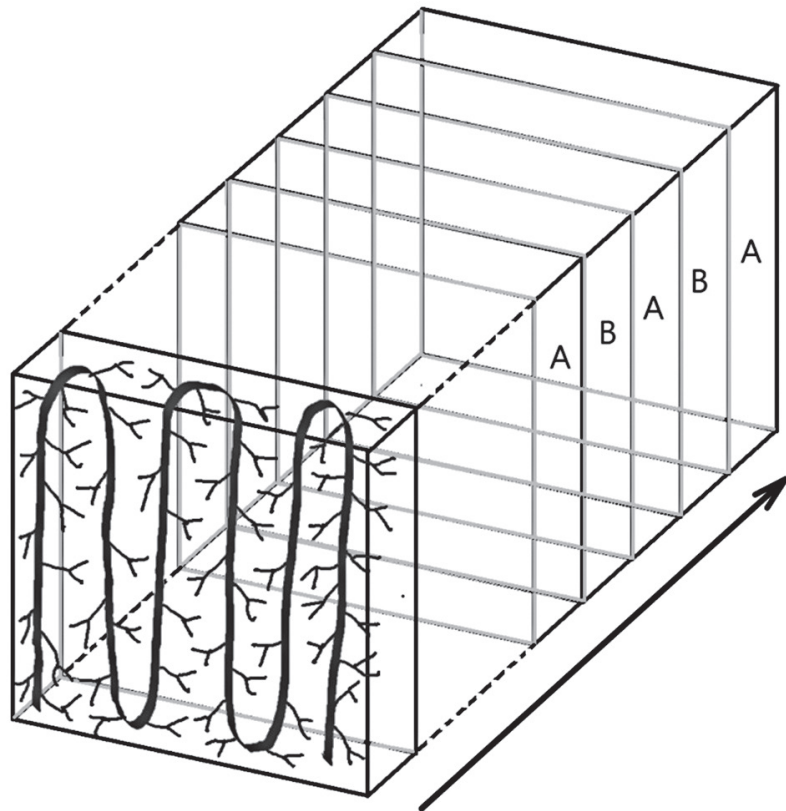

Figure 1. The polymer crystalline-like domain is constructed from many lamellar layers. The long polymer chain is periodically warped to like a paper clip in each layer and many functional groups (represented by $\mathrm{Y}$ shape here) link to the polymer chain. The direction of the arrow line represents the direction of the carrier transport. well as depolarization field from the "cavity effects" and in conductive polymers can reach values in tenths of eV. b) $J_{\alpha \beta}$ is the charge transfer integral between eigenstates $\alpha$ and $\beta$, which can be expressed as

$$
J_{\alpha \beta}=\Sigma_{n \in \mathrm{A}, m b \in \mathrm{B}} J_{n m} Z_{\alpha n} Z_{\beta m} \delta_{n m},
$$

where $n$ and $m$ are molecular site indexes in different polymer layers. The transfer integral in Eq. (4) is determined by the inter-chain transfer integral $J_{n m}$ between local sites $\left|c_{n}\right\rangle$ and $\left|c_{m}\right\rangle$ on adjacent polymer chains and $Z_{\alpha n} \equiv\left\langle\alpha \mid c_{n}\right\rangle$ are on-chain orbital expansion coefficients of the $\alpha$-th eigenstate into the basis of electronic states $\left|c_{n}\right\rangle$ located on individual repeat units (site representation). The expansion coefficient $Z_{\alpha n}$ needs to be calculated from the eigenvectors of Hamiltonian and its square value means the probability of finding a carrier residing in the $\alpha$-th state at the site $n$. In our simulation below we assume constant inter-chain transfer integrals, i.e., $J_{n m}=J$.

\section{Quantum states of the polymer chains}

We assume that the electronic levels are dominantly controlled by the following Hamiltonian [9].

$$
H=H_{e}+H_{h} .
$$

The first term in Eq. (5) $H_{e}$ describes the on-chain delocalization of electronic states as well the electronphonon coupling in the framework of the semi-classical SSH model leading to the effect of the Peierls instability, where in the stable configuration the bond lengths alter, and can be written as follows

$$
\begin{aligned}
& H_{e}=\Sigma_{n} \in_{0}\left|c_{n}\right\rangle\left\langle c_{n}\right|-\Sigma_{n} t_{n, n+1}\left(\left|c_{n+1}\right\rangle\left\langle c_{n}\right|+\text { h.c. }\right)+ \\
& +\frac{K_{s}}{2} \Sigma_{n}\left(u_{n+1}-u_{n}+C\right)^{2} .
\end{aligned}
$$

where $t_{n, n+1}=t_{0}-\alpha\left(u_{n+1}-u_{n}\right)+(-1)^{\mathrm{n}} t_{e}$ is the on-chain transfer integral between adjacent repeat units linearly dependent on their mutual distance $\left(u_{n+1}-u_{n}\right), \mathrm{t}_{0}$ and $\alpha$ are the interaction constants, $t_{e}$ describes alternation of $t_{n, n+1}$ arising from the alternation of the inter-unit distances $\left(u_{n+1}-u_{n}\right)$ due to Peierls instability; $\epsilon_{0}$ is the highest occupied orbital energy of the isolated $n$-th repeat unit, which can be varied by the applied bias voltage; $u_{n}$ is the displacement for the $n$-th molecule. The last term on the right side of Eq. (6) represents the spring potential with an effective spring constant $K_{s}$ and the equilibrium inter-unit distance $C$. The second term $H_{h}$ in Eq. (5) describes the hybridization coupling (charge transfer) between the polymer backbone and its side groups (or alternatively environmental states or additives)

$$
H_{h}=V_{p}\left(\Sigma_{n}\left|c_{n}\right\rangle\left\langle d_{n}\right|+\text { h.c. }\right)+E_{p}\left(\Sigma_{n}\left|d_{n}\right\rangle\left\langle d_{n}\right| .\right.
$$

In Eq. (7) $V_{p}$ determines energy of orbital hybridization (transfer integral) between states $\left|c_{n}\right\rangle$ and $\left|d_{n}\right\rangle$ 
located at the $n$-th repeat unit of the polymer backbone and its side group (substituent), respectively. The second term, $E_{p}$, represents the orbital energy of $\left|d_{n}\right\rangle$ with respect to $\epsilon_{0}$. We set it to be a constant for all $n$ sites.

Eigenstates $|\alpha\rangle$ of the Hamiltonian $\mathrm{H}$ (see Eq. (5)) and displacements $u_{n}$ are calculated self-consistently, while the latter are obtained from the minimum position $\partial E_{G} / \partial u_{n}=0$ of the total ground energy $E_{G} \equiv \Sigma_{\alpha}\langle\alpha|H| \alpha\rangle$ $f(\alpha\rangle$. In the last summation states $|\alpha\rangle$ are weighted by their Fermi-Dirac distribution $f(\alpha\rangle$. From this, we can determine $Z_{\alpha n} \equiv\left\langle\alpha \mid c_{n}\right\rangle$ needed for the estimation of the transfer integral (4), mean electronic densities $n_{c}$ and $n_{d}$ on polymer backbone and side groups, respectively, and also mean density of charge transfer $\Delta n \equiv n_{c}-n_{d}$ between polymer and linked group.

The model of charge transfer depicted above was formulated in the state space of electronic orbitals, the reformulation to the state space of holes is straightforward. For the polymers carrying $N$ sites and mean concentration $n_{h}$ of holes in the limit of half-filled band, the electronic densities $n_{c}$ and $n_{d}$ are coupled by the constraint $n_{c}+n_{d}=2-n_{h}$.

All the energy parameters in this paper are scaled in the unit of $\mathrm{eV}$, moreover, the units of the e-ph coupling constant $\alpha$ and the spring constant $K_{s}$ are $\mathrm{eV} / \AA^{2}$ and $\mathrm{eV} / \AA$, respectively. The values of the parameters are $t_{0}=1.5, t_{e}=0.1, \alpha=4.0$ and $K_{s}=15.0$. The former three parameters are chosen suitably for some general organic polymers and the relatively large value of $K_{s}$ was used to model the stiff polymers [9]. The number of molecular sites of a polymer chain in 30 and the number of lamellar layers in "the discrete box" is 20 . The distance $d$ between two nearest neighbour polymer layers is $3.85 \AA$ and the inter-chain transfer integral $J=0.01$ $\mathrm{eV}$, which is a characteristic value for such distances. For applied electric voltage both local on-chain energy $\epsilon_{0}$ and energy level $E_{p}$ of the state $\left|d_{n}\right\rangle$ shifts with the electric field by the same amount. For the energy level $E_{p}$ of attached groups we assume that it is of the order $0.2 \mathrm{eV}$ similarly as in Toman et al. [10]. For efficient charge transfer between monomer sites and side-groups, the values of the coupling interaction $V_{p}$ should be of comparable values. Throughout the model calculation we have assumed the room temperature.

\section{RESULTS AND DISCUSSION}

In our simulations we assumed that the electronic orbital levels of side groups $E_{p}>0$, so that these groups are electron donor (hole acceptor) states and for holes moving along the polymer backbone those side groups act as trap states. In the simulation below, the limiting hole concentration $n_{h} \rightarrow 0$ corresponds to the case of half-filled band as for the sites $\left|c_{n}\right\rangle$ and $\left|d_{n}\right\rangle$, respectively, just one unpaired electron is available independently of its spin orientation. The Peierls instability of the polymer chain creates a depletion of density of states (DOS) in the middle of the band, promoting thus a formation of an "effective" gap, however, linked sidegroups significantly promote formation of localized states close to the middle of DOS. Consequently, for the limit of the half-filled band, the Fermi level $E_{F}$ is close to those localized states and the "hole" mobility will be significantly influenced by the hybridization coupling here. In Figure 2 we studied dependence of calculated mobility on the on-chain site-side group hybridization coupling $V_{p}$ for low $n_{h}=0.03$ and mean $n_{h}=0.1$ hole concentration and for fixed value of the orbital energy $E_{p}=0.1 \mathrm{eV}$. We observe that the mobility provides a strong resonant amplification for relatively small coupling $V_{p} \sim 0.03 \mathrm{eV}$. This may be explained as a result of competition between two different processes. For the first, in the limit of very strong hybridization coupling

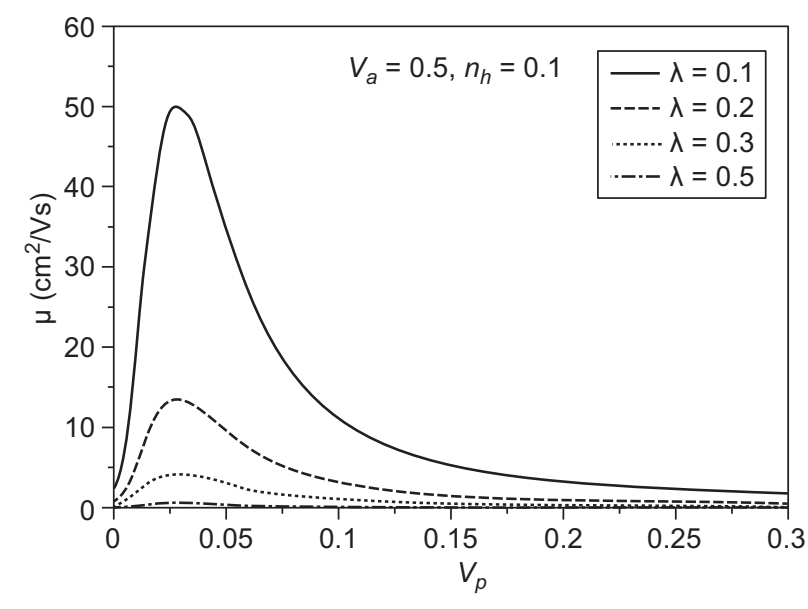

a)

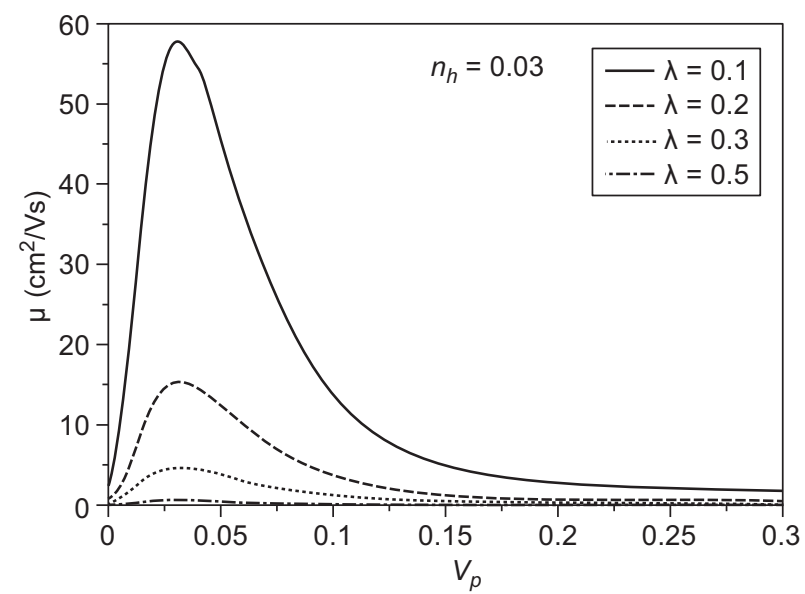

b)

Figure 2. The mobility dependence on the hybridization coupling $V_{p}$. The applied voltage $V_{a}=0.5 \mathrm{~V}$. The values of hole concentration $n_{h}=0.1$ (a), 0.03 (b), the reorganization energies $\lambda\left(\mathrm{in} \mathrm{eV}\right.$ ) are depicted in the figure. The orbital energy of side groups is $E_{p}=0.1 \mathrm{eV}$. 


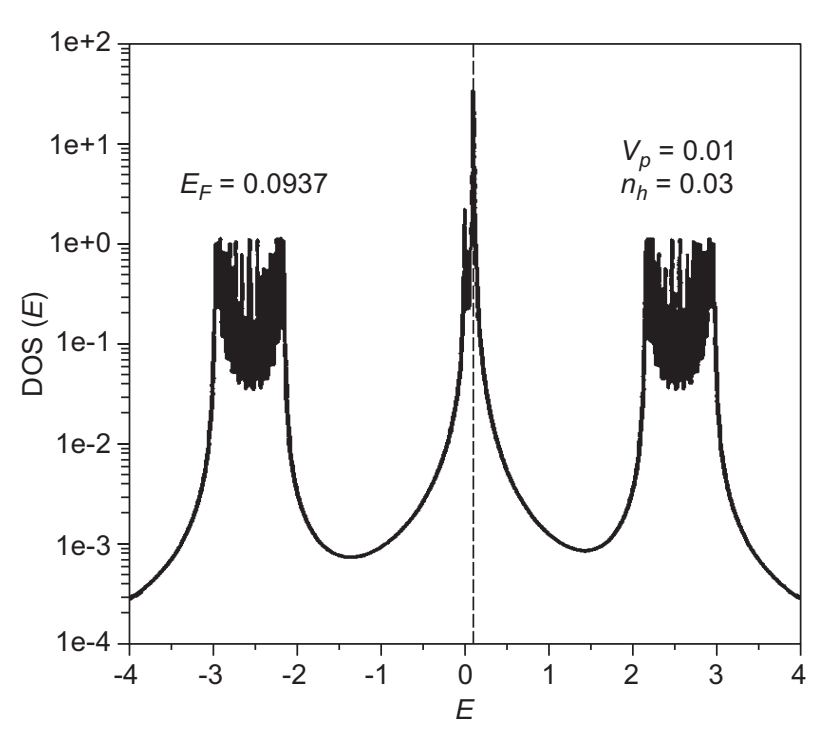

a)

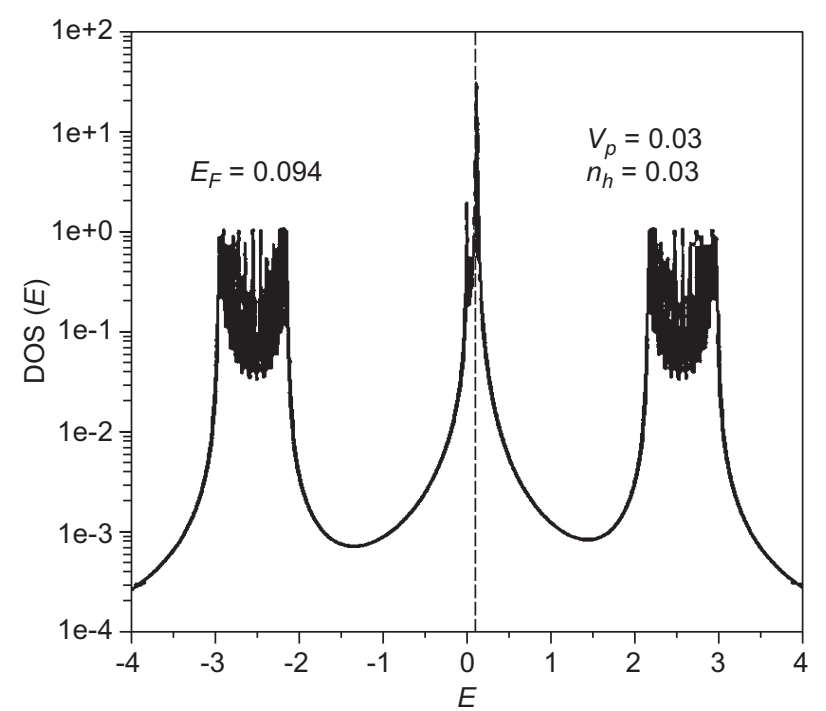

c)

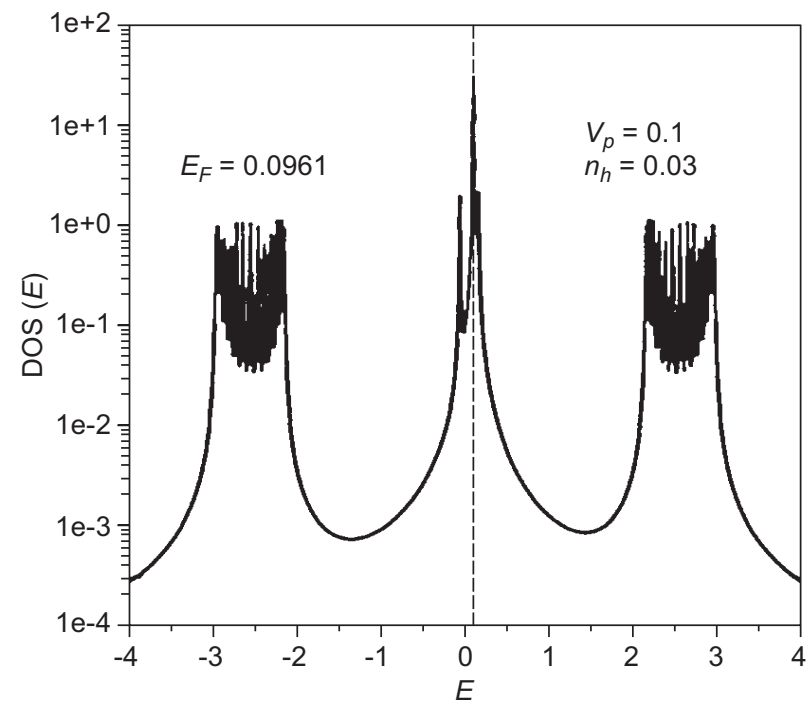

e)

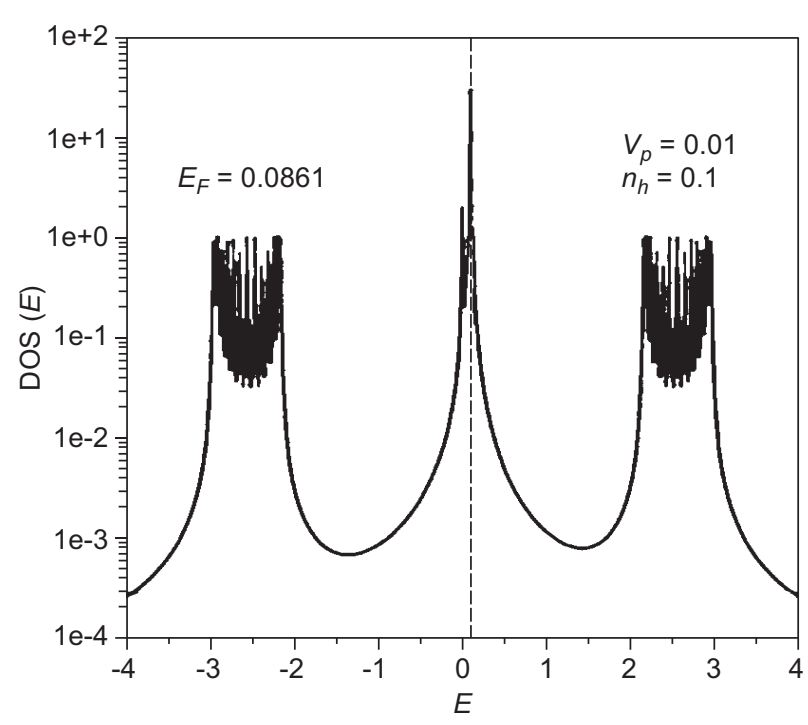

b)

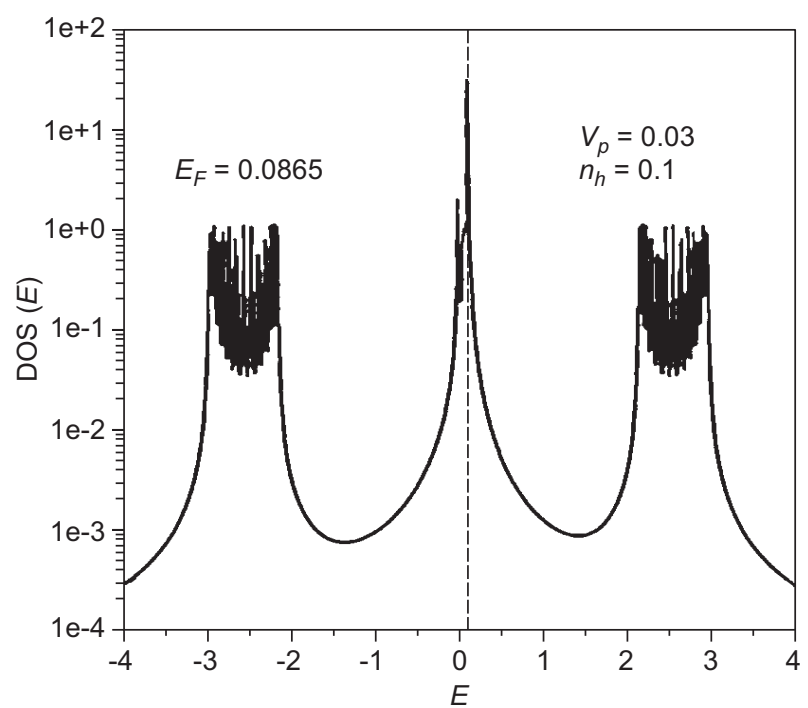

d)

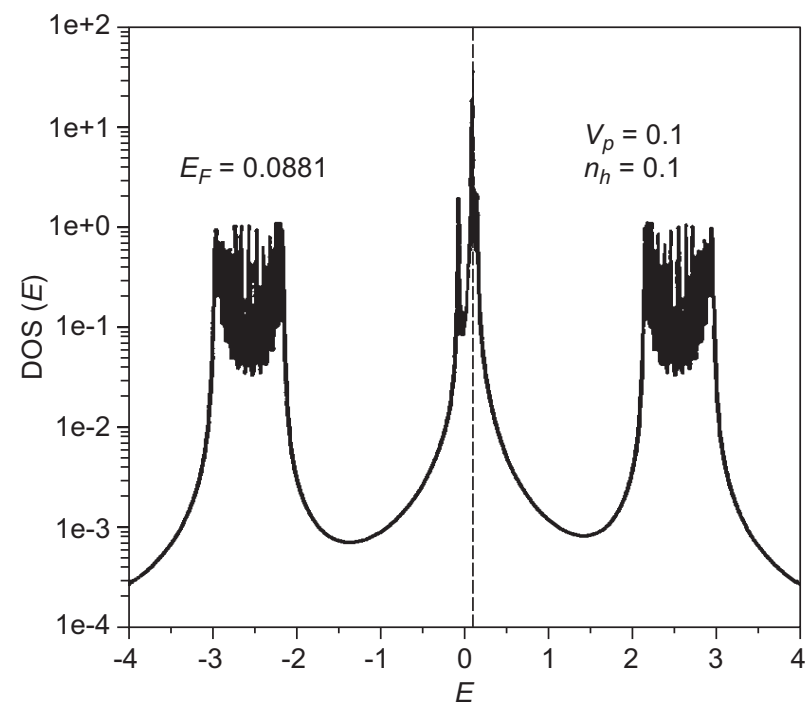

f)

Figure 3. Density of states calculated for different values of hybridization coupling $V_{p}$ and hole concentration $n_{h}$ (see legend). The orbital energies of linked groups are $E_{p}=0.1 \mathrm{eV}$. 
$V_{p}$, the electronic eigenstates $|\alpha\rangle$ near the middle of DOS are strongly localized to some local site, i.e., $|\alpha\rangle \rightarrow|K\rangle$. Then, $Z_{\alpha n} \sim \delta_{n K}$ and the transfer integral in (4) will be almost diagonalized $J_{\alpha \beta} \sim \delta_{\alpha \beta}$. The product $f\left(E_{\alpha}\right)\left(1-\left(E_{\alpha}\right)\right)$ in (2) takes maximum value at $f\left(E_{\alpha}\right)=1 / 2$, i.e, $E_{\alpha}=E_{F}$. Consequently, near Fermi level practically only charge transfer between corresponding energy level occurs, which significantly lowers the charge mobility. Additionally, increased hybridization coupling $V_{p}$ between the site $|K\rangle$ and the adjacent side group will increase their local energy splitting $\sqrt{ } E_{p}{ }^{2}+4 V_{p}^{2}$, which pushes Fermi level from charge transporting DOS (cf. Figure 3), which in turn will significantly decrease the charge transfer. On the other hand, for the limit of very weak hybridization coupling, the holes will be trapped in side-groups as $E_{p}>0$. In Figure 4 we studied the mobility dependence on the orbital energy $E_{p}$ of side-groups for fixed value of the hybridization coupling $V_{p}=0.1 \mathrm{eV}$. We observe that increase of the mobility happens for two different limiting values of the orbital energy $E_{p}$. For high values of $E_{p}$ the local dimer energy splitting increases (cf. Figure 5), but Fermi level also increases (gets closer to the orbital level $E_{p}$ ) as the DOS near Fermi level becomes asymmetrical and the transfer integral in (4) can mutually couple more states, which effectively increases the charge mobility. For the opposite limit of $E_{p} \rightarrow 0$ the "local dimer" energy splitting $\sim 2 V_{p}$ still exists, however, splitted ("+" and "-") levels have the same occurrence probability (projection coefficients) on the on-chain states so that both of them can participate in the inter-chain charge transfer. As for higher hole concentrations the overlap between DOS and probability of available states ("-“ levels) increases, we observe stronger increase of charge mobility for higher charge concentrations in the limit $E_{p} \rightarrow 0$ (cf. Figure 4). Another important information is to study the measure of charge transfer density $\Delta n=n_{c}-n_{d}$ between on-chain sites and linked groups as this quantity controls the mean hole density $1-n_{c}$ on the polymer backbone by the relation $1-n_{c}=\left(n_{h}-\Delta n\right) / 2$. In Figure 6 we studied the dependence of $\Delta n$ on the hybridization coupling $V_{p}$ (for fixed $E_{p}=0.1 \mathrm{eV}$ ) and orbital energy $E_{p}$ (for fixed $V_{p}=0.1 \mathrm{eV}$ ) at various hole concentrations $n_{h}$. We observed that for zero hole concentration the mean hole concentration on the polymer backbone $1-n_{c}=\left(n_{h}-\Delta n\right) / 2<0$, i.e., holes are trapped on side-groups for both dependences and the backbone is negatively charged. By careful inspection in Figure 6 we observe that for non-zero hole concentration such "negatively charged backbone" $\left(\Delta n>n_{h}\right)$ appears for higher values $E_{P}$ and lower values of $V_{p}$. This not necessarily means that for such cases the layers are "no longer hole transporting", because such "negatively charged polymer backbones" appear equivalently on adjacent polymer lamellas, where the Fermi levels are increased in the same way. However, for the case of $n_{h}=0.1$ we see (cf. Figure 6 , left) that for $V_{p}<0.05 \mathrm{eV}$ the backbone becomes negative $\left(\Delta n>n_{h}\right)$ and for lower values of $V_{p}$ this "negativity of the polymer backbone" increases and holes are trapped in the linked groups. This is correlated with very stronger decay of mobility for very small hybridization coupling in Figure 2. In Figure 6, left, we can also notice that for small values of $V_{p}$ the backbone is "negative" $\left(\Delta n>n_{h}\right)$ for all hole concentrations, while for higher values of $V_{p}$ the backbone is "negative" $\left(\Delta n>n_{h}\right)$ for lower hole concentrations and becomes "positive" $\left(\Delta n<n_{h}\right)$ for higher hole concentration, because linked groups are filled with holes. This is very correlated with profiles of mobility dependences on hole concentration shown in Figure 7 where we observe that for weak coupling $V_{P}$ the mobility only slightly decreases with hole concentrations, while for higher values of $V_{P}$ the mobility increases up with hole concentration up to some

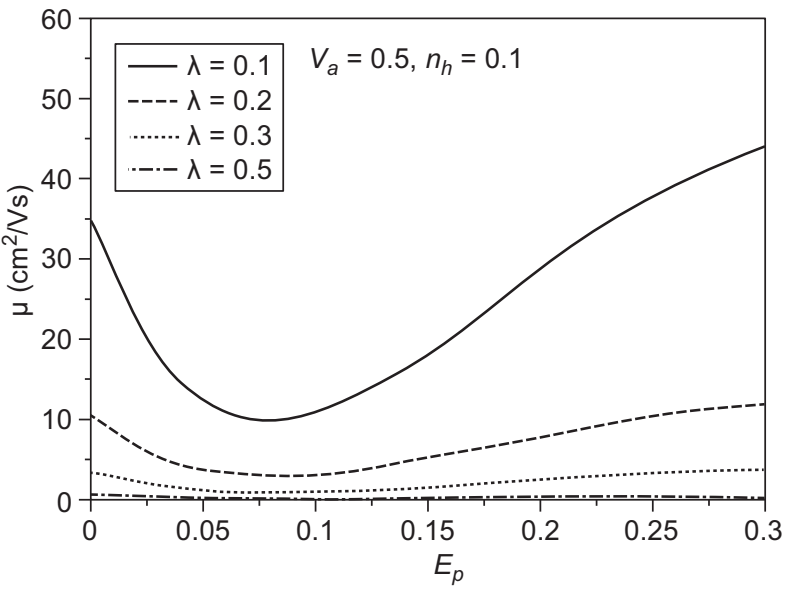

a)

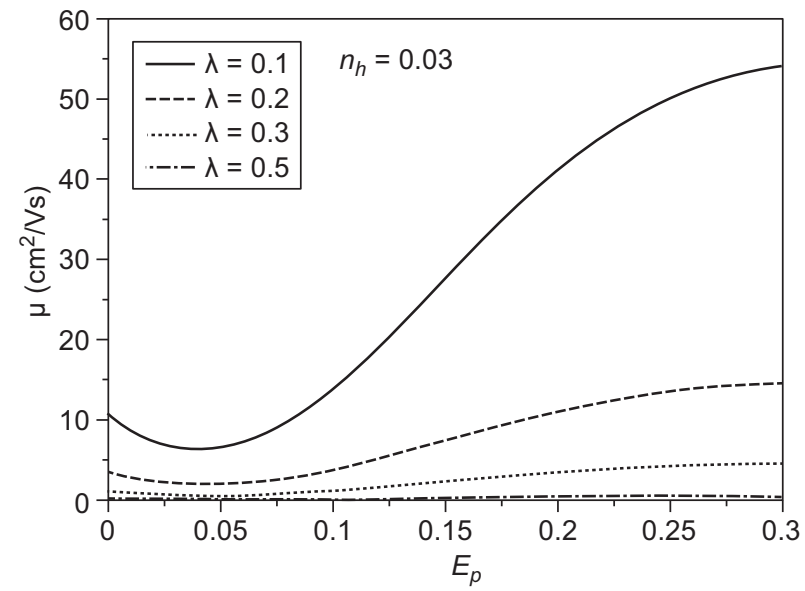

b)

Figure 4. The mobility dependence on the orbital energy of linked groups $E_{p}$ calculated for different reorganization energies $\lambda$ (see legend). The applied voltage $V_{a}=0.5 \mathrm{~V}$, the values of hole concentration $n_{h}=0.1$ (a) and $n_{h}=0.03$ and the hybridization coupling $V_{p}=0.1 \mathrm{eV}$. 


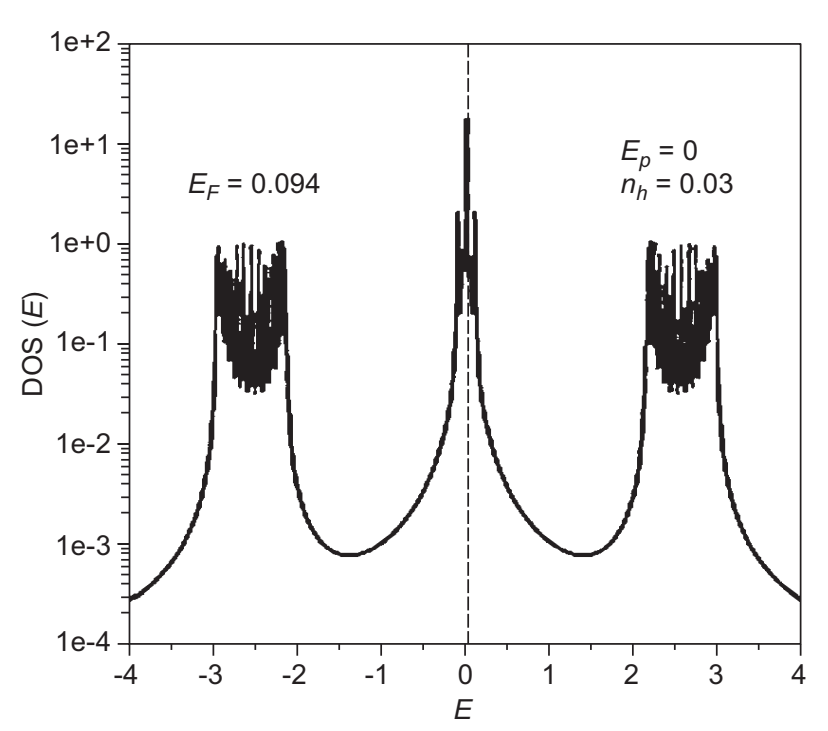

a)

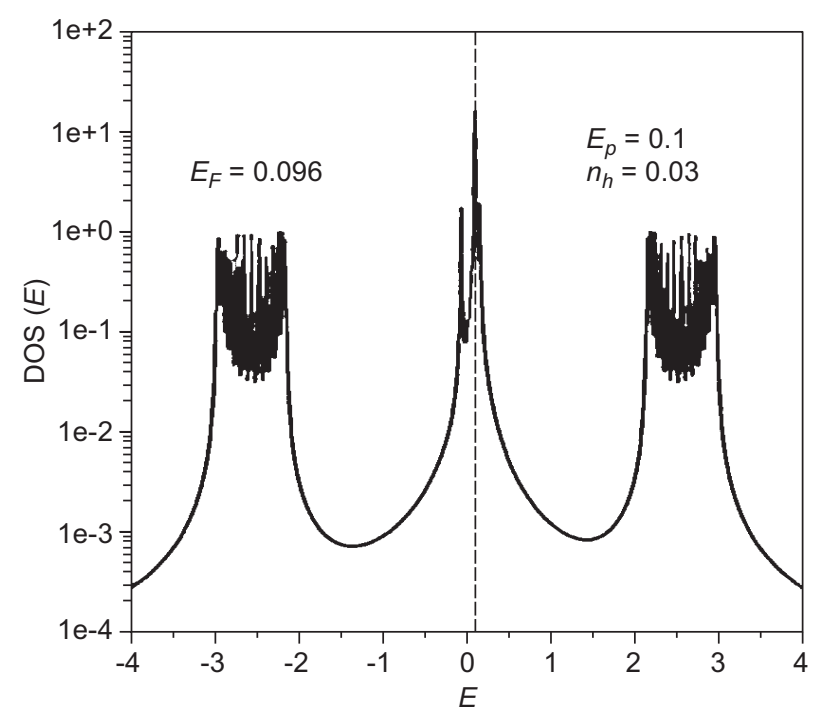

c)

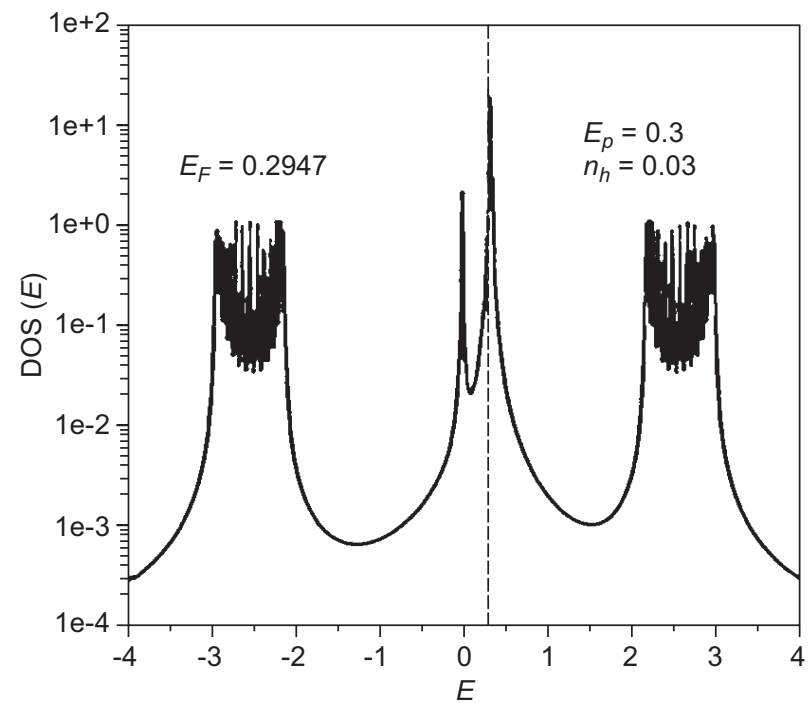

e)

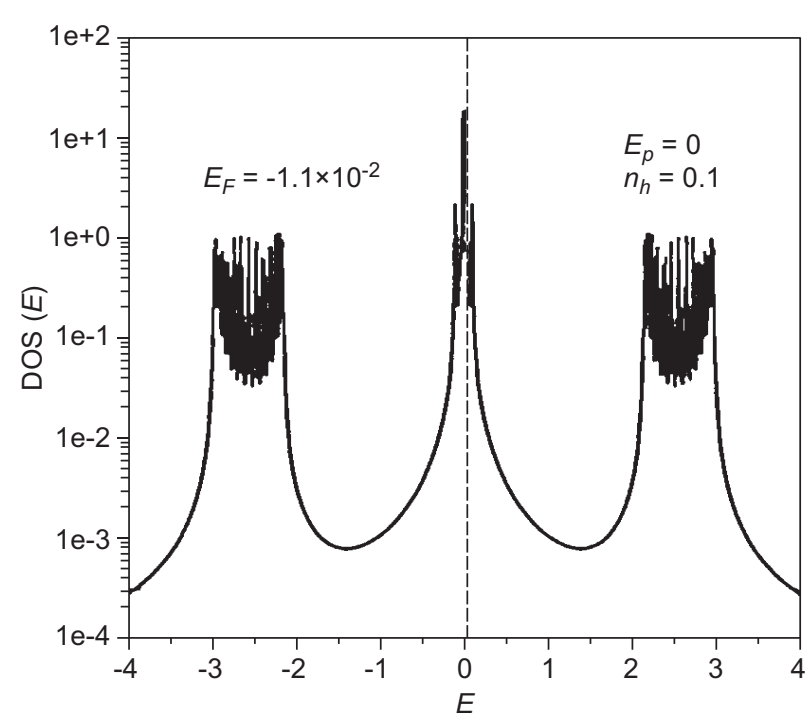

b)

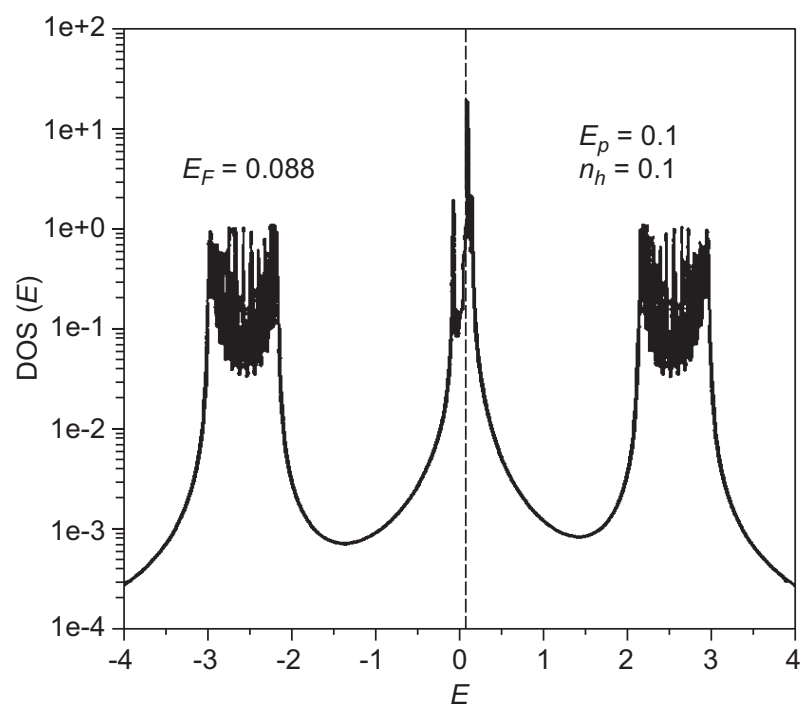

d)

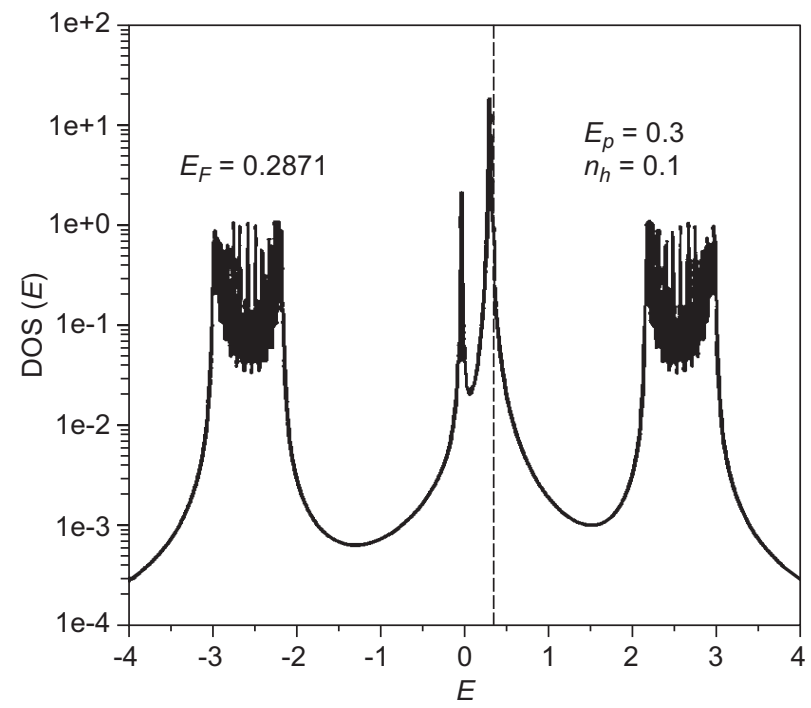

f)

Figure 5. Density of states calculated for different values of energies $E_{p}$ of side groups and hole concentration $n_{h}$ (see legend). The hybridization coupling $V_{p}=0.1 \mathrm{eV}$. 
critical value due to the trap-filling and then it decreases for high hole concentration due to the Pauli exclusion principle. Generally, lower mobilities for higher values of the hybridization coupling $V_{P}$ were discussed above due to the effect of the on-chain charge localization accompanied by the reduction of charge transfer between corresponding energy levels. We also note slight "jags" in the mobility dependence, which are related to the discrete structure of density of states and its partially asymmetrical distribution. As the charge concentration gets to some critical value, the contribution of a particular level to the mobility can be promoted. However, the relative deviations in mobility due to these "jags" are less than 5 percent.

\section{CONCLUSIONS}

We have studied mobility dependences of the transversal hole transfer between stacks of conductive polyacetylene-like polymer lamellas in the limit of transition from the conductive to semiconductive phase due to the Peierls instability induced by the electronphonon coupling. The model also assumed hybridization coupling $V_{p}$ of local monomer sites to linked groups with positive orbital energy $E_{p}$ with respect to the monomer units of the polymer backbone. We have found that the charge mobility:

- resonantly increases with small values of hybridization coupling $V_{p}$

- takes minimum value for some "moderate" value of the orbital energy $E_{p}$ of linked group, but increases for high and low values of $E_{p}$.

- slightly decreases with hole concentration for low values of hybridization coupling $V_{p}$, but takes maximum at some critical hole concentration for strong hybridization coupling $V_{p}$.

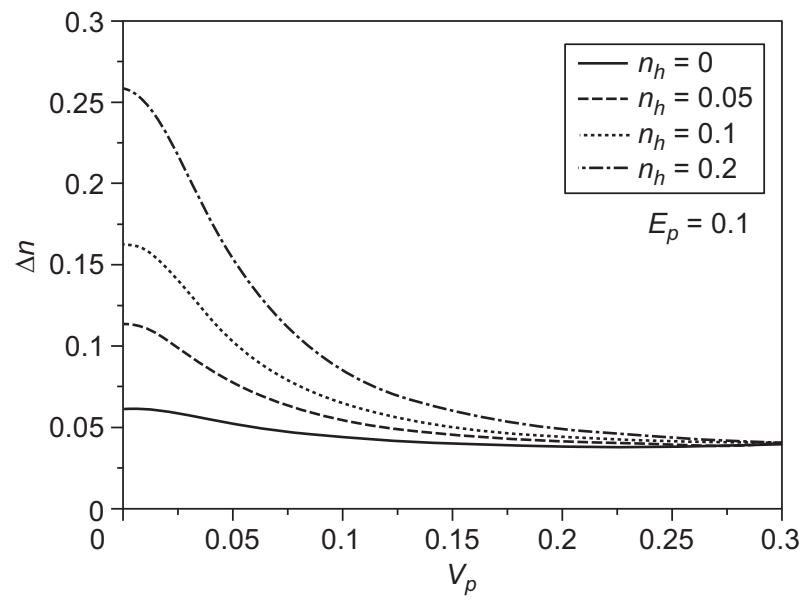

a)
We find those conjectures important for chemistry design of organic optoelectronic devices as it provides an important insight to the problem how the hybridization coupling of the polyacetylene-like polymers controls the hole mobility. Namely, we have proved very strong sensitivity to those couplings.

\section{Acknowledgement}

The work was funded by the Ministry of Science and Technology of Taiwan (project No. MOST 104-2112-M390-001 (Shih-Jye Sun)), Ministry of Education, Youths and Sport of the Czech Republic (project No. LD 14011 under COST Action MP1202-HINT), Czech Science Foundation (project No. 15-05095S) and Czech Academy of Sciences (project CAS KONNECT-007).

\section{REFERENCES}

1. Yamashita Y. (2009): Organic semiconductors for organic field-effect transistors. Science and Technology of Advanced Materials, 10, 024313.

doi:10.1088/1468-6996/ 10/2/024313

2. Kang I, Yun H.J., Chung D.S., Kwon S.K., Kim Y.H. (2013): Record high hole mobility in polymer semiconductors via side-chain engineering. Journal of American Chemical Society, 135, 14896-14899. doi: $10.1021 / \mathrm{ja} 405112 \mathrm{~s}$

3. Salleo A. (2015): Organic electronics: Something out of nothing. Nature Materials, 14, 1077-1078. doi: $10.1038 /$ nmat4420

4. Boufflet P., Han Y., Fei Z., Treat N.D., Li R., Smilgies D.M., Stingelin N., Anthopoulos T.D., Heeney M. (2015): Using molecular design to increase hole transport: backbone Fluorination in the Benchmark Material Poly(2,5-bis(3-alkylthiophen-2-yl)thieno[3,2-b]-thiophene (pBTTT). Advanced Functional Materials, 25, 7038-7048. doi: 10.1002/adfm. 201502826

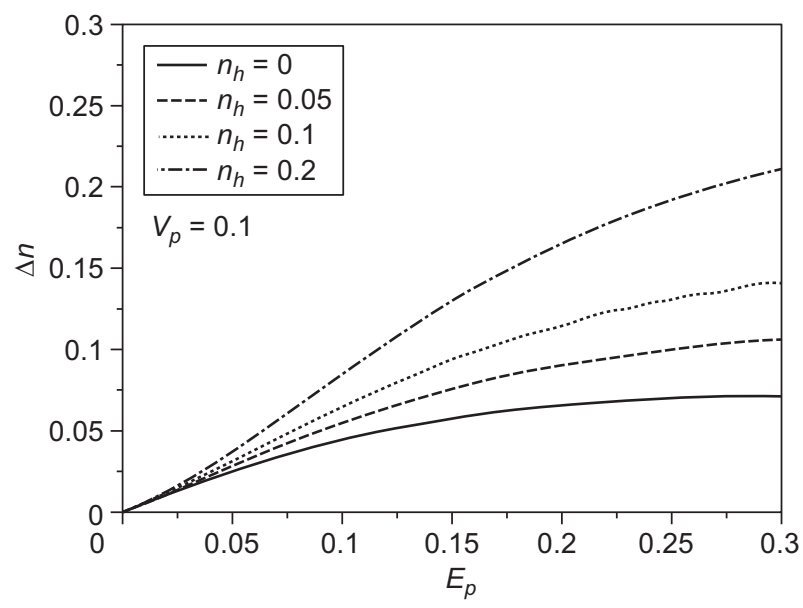

b)

Figure 6. The dependence of mean density $\Delta \mathrm{n}$ of charge transfer on hybridization couplings $V_{p}$ (a) and orbital energy of linked group $E_{p}(\mathrm{~b})$. The hole concentration $n_{h}$ are shown in the figure legend. 




a)

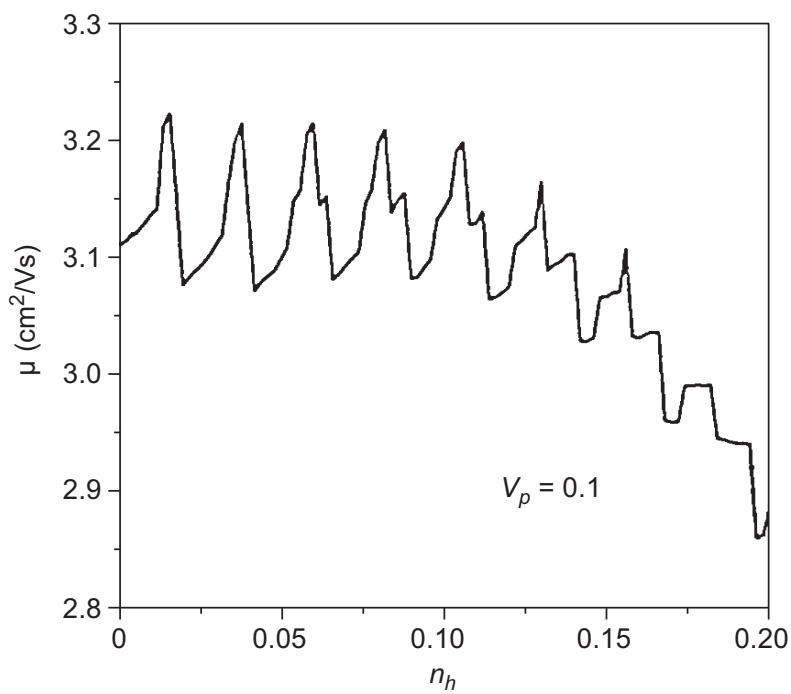

c)

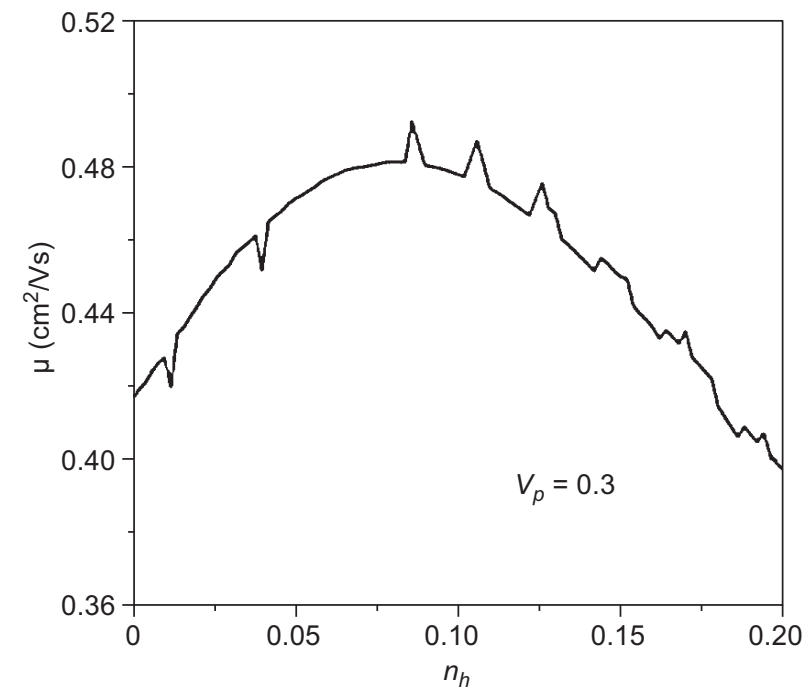

e)



b)

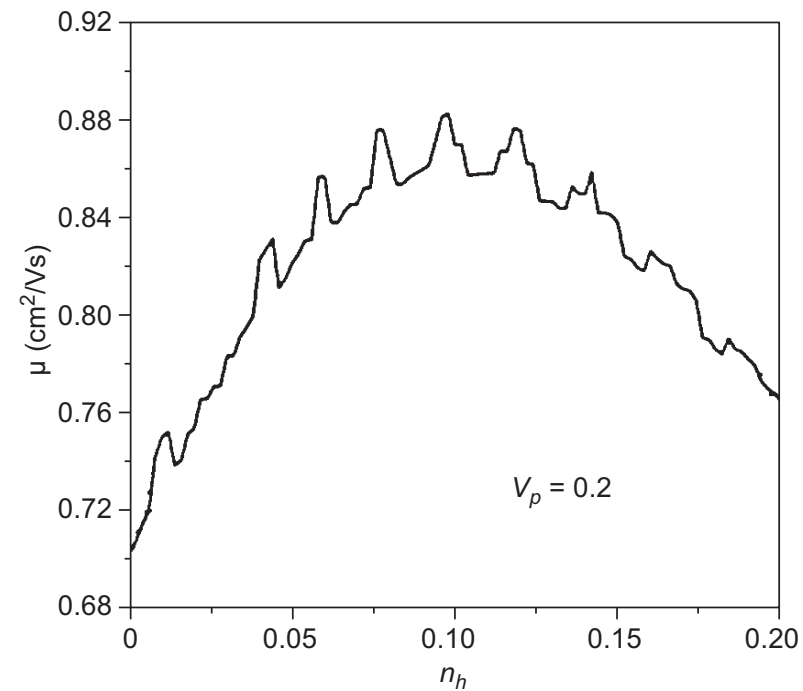

d)

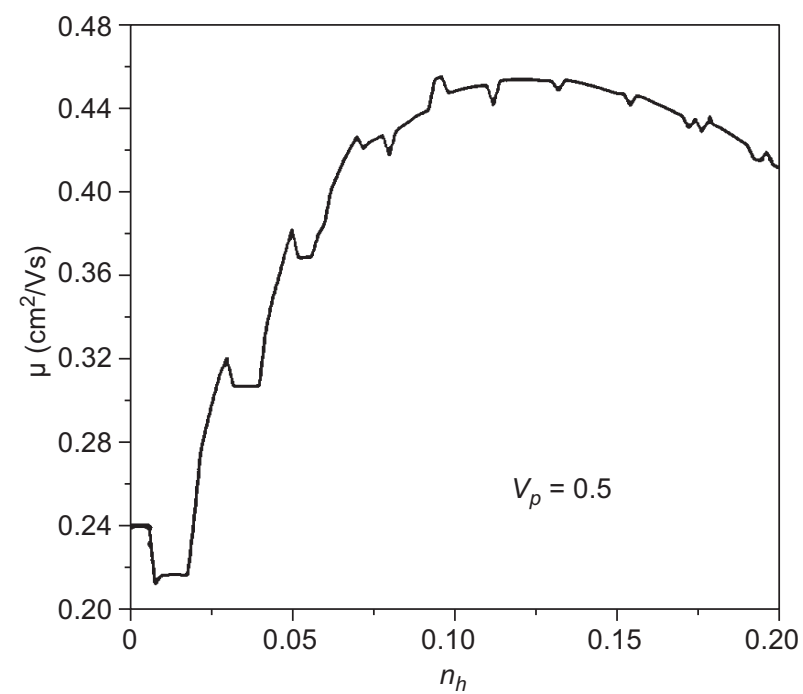

f)

Figure 7. The mobility dependence on the hole concentration $n_{h}$. The orbital energies of linked groups are $E_{p}=0.1 \mathrm{eV}$, the applied voltage $V_{a}=0.5 \mathrm{~V}$ and the reorganization energy $\lambda=0.2 \mathrm{eV}$. The hybridization coupling $V_{p}$ varies from the weak up to the strong limit as shown in figure legend. 
5. Noriega R., Rivnay J., Vandewal K., Koch F.P., Stingelin N., Smith P., Toney M.F., Salleo A.R., (2013): A general relationship between disorder, aggregation and charge transport in conjugated polymers. Nature Materials, 12, 1038-1044. doi: 10.1038/nmat3722

6. Toman P., Nešpůrek S., Bartkowiak W., (2009): Modelling of charge carrier transport in conjugated polymers doped by polar additives. Material Science-Poland, 27, 797-812

7. Silvestri F., Marrochi A., (2010): Acetylene-based materials in organic photovoltaics. International Journal of Molecular Sciences, 11, 1471-1508. doi:10.3390/ijms11041471
8. Su W.P., Schrieffer J.R., Heeger A.J. (1979): Solitons in polyacetylene. Physical Review Letters, 42, 1698-1701. doi: 10.1103/PhysRevLett.42.1698

9. Sun S.J., Lin C.Y., Yu C.F., (2011): Transport properties of orbitally hybridized organic semiconductors. The European Physical Journal B, 83, 173-179. doi: 10.1140/epjb/e2011-20253-20254

10. Toman P., Bartkowiak W, Nešpůrek S., Sworakowski J., Zalesny R., (2005): Quantum-chemical insight into the design of molecular optoelectrical switch. Chemical Physics, 316, 267-278. doi: 10.1016/j.chemphys.2005.05.028 